\title{
Selección de residentes en la formación de psiquiatría: ¿qué atributos buscan los docentes de un centro formador y por qué medios?
}

\author{
Resident selection in psychiatry: what attributes are sought and by what means? \\ Bernardo Pacheco Pacheco', Francisca Valdés Rojicํㅜ, Valentina Kobus Garín², Paula Bedregal García ${ }^{3}$
}

\section{Resumen}

Introducción: la formación en Psiquiatría tiene como primer desafío elegir los candidatos ideales para desempeñarse óptimamente como residentes, alcanzando las competencias necesarias para un posterior ejercicio de la especialidad. En general, no se cuenta con instrumentos específicos de selección de residentes, utilizándose diversas estrategias de elección de candidatos a la especialidad. El objetivo del presente estudio es identificar los atributos y los medios de selección que un grupo de académicos consideran necesarios al momento de seleccionar a los candidatos a sus programas de residencia en Psiquiatría.

Metodología: se realizó una investigación cualitativa de tipo encuesta de percepción utilizando el Método Delphi. La encuesta se envió, por medio de una plataforma web, a todos los académicos del Departamento de Psiquiatría de la Pontificia Universidad Católica de Chile $(n=39)$.

Resultados: los atributos mencionados fueron agrupados en 8 dominios. Las principales categorías identificadas por los encuestados fueron: profesionalismo (41\%), motivación por la especialidad (33\%), habilidades de comunicación (13\%) y estabilidad psicológica (12\%). El principal instrumento mencionado para evaluar los atributos de los postulantes fue la entrevista personal. Las publicaciones científicas, las notas de pregrado y las cartas de recomendación son mencionadas secundariamente.

Conclusiones: el actuar profesional en la acción médica, medido como profesionalismo, en este estudio es atributo esencial en un postulante a un programa de residencia en Psiquiatría. Aunque las entrevistas personales han demostrado poco valor predictivo con el rendimiento del residente durante su formación, siguen siendo un instrumento muy requerido en los procesos de selección de residentes.

Palabras clave: selección de residentes; entrevista personal; competencias clínicas.

\begin{abstract}
Introduction: Training in Psychiatry has, as its first challenge, to choose the ideal candidates to perform optimally as residents, reaching the necessary skills for a subsequent exercise of the specialty. There are no specific instruments for selecting residents, but many different strategies are used to select proper candidates. The primary objective of this study is to recognize the attributes academics seek in a psychiatry residency applicant. The secondary objective was to identify the selection method academics thought as optimal. Methods: A qualitative investigation of perception was conducted using the Delphi Method. The perception survey was sent through a web platform to all academics of the Department of Psychiatry of the Pontificia Universidad Católica de Chile $(n=39)$.

Results: Attributes were grouped into eight domains. The main identified categories were: professionalism (41\%), specialty interest (33\%), communication skills (13\%), and psychological balance (12\%). A personal interview was seen as the current optimal selection method. Publications, undergraduate scores, and letters of recommendation were also taken into account.

Conclusions: Professionalism, is seen as an essential attribute in an applicant for a psychiatry residency program. Although personal interviews have shown little predictive value related to the resident's performance during their training, they are still a very common and valued instrument used in the resident selection processes.
\end{abstract}

Keywords: resident selection; personal interview; clinical skills.

Fecha de envío: 29 de julio de 2019 - Fecha de aceptación: 13 de marzo de 2020

(1) Departamento de Psiquiatría, Pontificia Universidad Católica de Chile.

(2) Facultad de Medicina, Pontificia Universidad Católica de Chile.

(3) Departamento de Salud Pública, Pontificia Universidad Católica de Chile.

Autor de correspondencia: bejupa@vtr.net 


\section{Introducción}

La formación de médicos especialistas es un proceso que implica una gran inversión de recursos para las instituciones formadoras, como también dedicación y tiempo por parte de los cuerpos docentes. El objetivo final de una formación médica exitosa es aportar a la sociedad profesionales idóneos dispuestos a la atención de las necesidades de salud de la población (Jaramillo \& Martín, 2012). Por lo tanto, un proceso de selección de candidatos a especialistas debería ser eficaz en identificar los postulantes que mejor se desempeñarán en un programa de formación, así como en la práctica médica futura. Dicho desempeño puede establecerse en distintos términos, en mejor rendimiento académico y teórico, o bien, mejores aptitudes y características no académicas de la persona del postulante (por ejemplo, aspectos profesionales, éticos, comunicacionales, etc.). Estos objetivos de selección son los que las distintas estrategias utilizadas buscan identificar. Sin embargo, ya sea para el desempeño académico como el no académico, hay poco consenso en cuanto a cuáles son los atributos del postulante, los instrumentos o las estrategias de selección más útiles para seleccionar adecuadamente a los futuros especialistas (Harfmann \& Zirwas, 2011). Múltiples estudios realizados sobre el tema no han sido concluyentes en definir cuál es el mejor método (Hamdy et al., 2006; Kenny et al. 2013).

A nivel internacional, las estrategias de selección varían. Se han estudiado las opiniones de los directores de programas de residencia sobre la importancia relativa de las diversas estrategias de selección (Wagoner \& Suriano, 1992; Green et al., 2009; Hartman, 2019). En varias especialidades, las tres estrategias de selección más consideradas fueron las calificaciones de pasantías prácticas ("clerkships"), las cartas de recomendación y los resultados de la prueba de conocimientos teóricos (como el Examen de Licencia Médica de los EE.UU, USMLE por sus siglas en inglés) (Prober et al., 2016; Wagner, 2017). EI USMLE ha presentado mayor validez predictiva de rendimiento futuro del residente (Berner, 1993), aunque solo para el primer año de residencia en algunos casos (Smilen, 2001). La experiencia en investigación y las cartas de recomendación, al igual que las entrevistas, han tenido poca asociación con un mejor rendimiento durante la residencia, sin embargo, son frecuentemente consideradas por los comités de selección (Kenny et al., 2013). En Europa, se distinguen globalmente dos modelos de selección (Lorusso \& González, 2013). El modelo mediterráneo, aplicado en Francia, España, Italia, Portugal, Rumania y Grecia, el cual utiliza una prueba escrita administrada a nivel nacional o regional que consiste en un test teórico con preguntas de selección múltiple y respuesta única. En algunos países se agrega una simulación escrita de caso clínico y se pondera de modo particular el expediente académico de presentación. En el modelo anglosajón, aplicado en el Reino Unido, Suecia, Alemania, Austria, Bélgica, Irlanda, Países Bajos, Polonia y República Checa, se considera preferentemente la solicitud del candidato y la entrevista personal; los exámenes nacionales de conocimiento teórico no son prioritarios (Lobato et al., 2015).

En nuestro país, la principal modalidad para acceder a un programa de especializaciones mediante los distintos concursos que realiza el Ministerio de Salud (MINSAL). Complementario a esto, cada Universidad realiza su propio proceso de habilitación que actúa como mecanismo seleccionador de los postulantes. Existe una minoría de postulantes, que acceden a los programas de formación de especialidades mediante concursos o procesos particulares gestionados por las mismas instituciones formadoras. Estos establecen sus propios requisitos y procesos de selección en concordancia con lo solicitado en los concursos ministeriales.

En términos generales, existen el Concurso Nacional de Ingreso al Sistema Nacional de Servicios de Salud (CONISS) y la Etapa de Destinación y Formación (EDF). En el primero, el médico recientemente egresado postula según ranking a un programa de residencia financiado por algún Servicio de Salud. En el segundo, el médico recién egresado trabaja inicialmente como médico general por un periodo de 3 a 6 años, para luego postular, también por ranking, a un programa de residencia en algún centro formador. (Clouet-Huerta et al., 2017; MINSAL, 2019).

En general, en el proceso de selección de residentes de especialidades médicas, algunos de los instrumentos de selección más usadas han sido: el rendimiento y la experiencia durante una rotación clínica optativa de la especialidad a la que se postula; el puntaje obtenido en pruebas nacionales de conocimientos teóricos; las notas obtenidas y publicaciones durante los años de pregrado; el desempeño en entrevistas personales; el ranking de pregrado; cartas de recomendación; participación en investigación y publicaciones científicas, y actividades extracurriculares, entre otras (Egol et al., 2011; Jaramillo \& Martín, 2012; Kenny et al., 2013, Agarwal., et al 2018).

Se ha observado que la entrevista personal es el instrumento de selección que ha sido más ampliamente utilizado en la postulación a programas de residencia (Stephenson et al., 2015). Algunos destacan como positivo que, tanto los encargados de la selección como los postulantes tienen una opinión positiva del uso de entrevistas; consideran éstas permiten la evaluación de habilidades comunicacionales, madurez personal, interés por la especialidad, honestidad entre otros aspectos (Wagoner et al., 1986). Contrariamente, otros estudios indican que no sería un método ideal de selección debido a que es difícil de estandarizar y que tiene 
escasa asociación con el rendimiento del residente y la práctica futura de la especialidad (Kenny et al., 2013). Se ha cuestionado, además, su utilidad en el caso de que los entrevistadores conozcan personalmente a los postulantes previo a las entrevistas (Smilen \& Bianco, 2001), y también cuando los antecedentes académicos del postulante son conocidos previamente antes de la realización de ésta (Illahi, 2017)). En ambos casos se correría el riesgo de sesgar la elección reduciendo la objetividad del procedimiento.

El surgimiento de los programas basados en competencias, como el modelo canadiense CanMEDS (CanMEDS, 2019), ha estimulado que la selección de residentes sea llevada a cabo por comités de selección de residentes que definan previamente que atributos esperan encontrar en los postulantes a especialidades, de modo que, las cualidades seleccionadas se ajusten al currículum del programa y a lo que cada especialidad médica defina como médico especialista experto. El desafío para el cuerpo docente es poder definir y predecir anticipadamente el potencial de aprendizaje de cada residente para una determinada especialidad. La Escuela de Posgrado de la Facultad de Medicina de la Pontificia Universidad Católica de Chile (PUC) desde el año 2015 ha asumido el modelo educacional basado en competencias CanMEDS en la formación de sus especialistas. Es así que las especialidades de Psiquiatría general de adultos e infanto juvenil de esta casa de estudios han ajustado sus programas de formación siguiendo los parámetros de la formación de especialistas basado en competencias. Los docentes académicos del Departamento de Psiquiatría participan seleccionando candidatos para la especialidad mediante comités de selección de residentes.

El objetivo del presente trabajo fue responder a las siguientes preguntas de interés docente: ¿cuáles son los atributos que los docentes seleccionadores de residentes creen que deben estar presentes en los candidatos a la especialidad de Psiquiatría? y, ¿con qué herramientas o instrumentos de selección estos docentes evalúan la presencia de tales atributos en los postulantes a la residencia?

\section{Metodología}

Para conocer la opinión de los docentes encargados de seleccionar a los candidatos a la especialidad de Psiquiatría se optó por una investigación cualitativa utilizando el Método Delphi, partiendo con la confección de una encuesta de percepción (Linstone \& Turoff, 2002). Esta técnica de producción de datos corresponde a un proceso de comunicación grupal en que se selecciona un grupo de expertos a los que se le pregunta su opinión respecto a un tema en particular. Las estimaciones de los expertos se obtienen en rondas sucesivas, anónimas, con el fin de tratar de conseguir convergencia y consenso respecto de la cuestión consultada y con la máxima consideración de las opiniones de cada uno de los participantes. Las encuestas de percepción se enviaron a través de una plataforma web a todos los académicos del Departamento de Psiquiatría PUC. El grupo de académicos docentes estuvo compuesto por psiquiatras, psicólogos e investigadores $(n=39)$. En la primera ronda de la encuesta se le solicitó a cada uno que mencionara los principales atributos que considera importantes al momento de seleccionar a un postulante para las residencias de Psiquiatría. Las respuestas recibidas se categorizaron por dominios. En una segunda ronda (búsqueda de consenso), se les preguntó a los académicos su grado de acuerdo o desacuerdo con los resultados que tanto ellos como los demás académicos habían respondido en la primera ronda. De este modo, se buscó en ellos estimular una nueva elección de atributos, o bien, una afirmación de los mencionados anteriormente. En esta segunda ronda también se les preguntó qué instrumentos o medios utilizarían para evaluar la presencia de los atributos esperados por ellos en el postulante. Los resultados de ambas fases fueron tratados utilizando una plataforma online de análisis y respuestas con metodología estadística básica.

Este estudio contó con la aprobación del Comité de Ética de la Facultad de Medicina de la PUC (Proyecto № 16-314). En el año 2017 fue presentado en modalidad poster en el Congreso Latinoamericano en Educación de Residentes (LACRE).

\section{Resultados}

Las respuestas de la primera ronda ( $100 \%$ de los participantes) fueron agrupadas en 8 dominios: profesionalismo, motivación por la especialidad, estabilidad psicológica, habilidades comunicacionales e interpersonales, excelencia académica, capacidad de trabajo en equipo, inteligencia e interés científico. En la segunda fase se buscó llegar a un consenso entre los académicos encuestados, contrastando las opiniones emitidas en la primera fase con posibles nuevas respuestas. En esta misma fase, los académicos fueron informados de los dominios construidos y se les preguntó por el grado de acuerdo respecto a estos. Del total de los académicos, sólo 26 de ellos (67\%) respondieron satisfactoriamente la segunda fase. La figura 1 muestra los 8 dominios con sus respectivos grados de acuerdo: muy de acuerdo (MDA), de acuerdo (DA) y en desacuerdo (ED). "Profesionalismo"fue el principal dominio en que se agruparon los atributos mayormente mencionados por los académicos. Los dominios "motivación por la especialidad", "estabilidad psicológica" $y$ "habilidades comunicacionales e interpersonales" estuvieron en segundo lugar. Los dominios"interés científico"e "inteligencia"fueron los menos votados por los encuestados en grado de importancia positiva (MDA) al momento de seleccionar un residente. La figura 2 muestra el grado de acuerdo global, (MDA+DA) para los distintos domi-

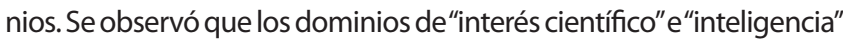
fueron mejor considerados en importancia positiva (MDA3 + DA18 
= 21 menciones; MDA6 + DA15= 21 menciones, respectivamente). Los dominios "excelencia académica" y "capacidad de trabajo en equipo" obtuvieron cada uno el mismo grado de acuerdo global
(MDA 14 menciones + DA11 menciones), quedando distantes a los 2 dominios de mayor acuerdo (MDA): "profesionalismo" (MDA 24 menciones) y"motivación por la especialidad"(MDA 22 menciones).

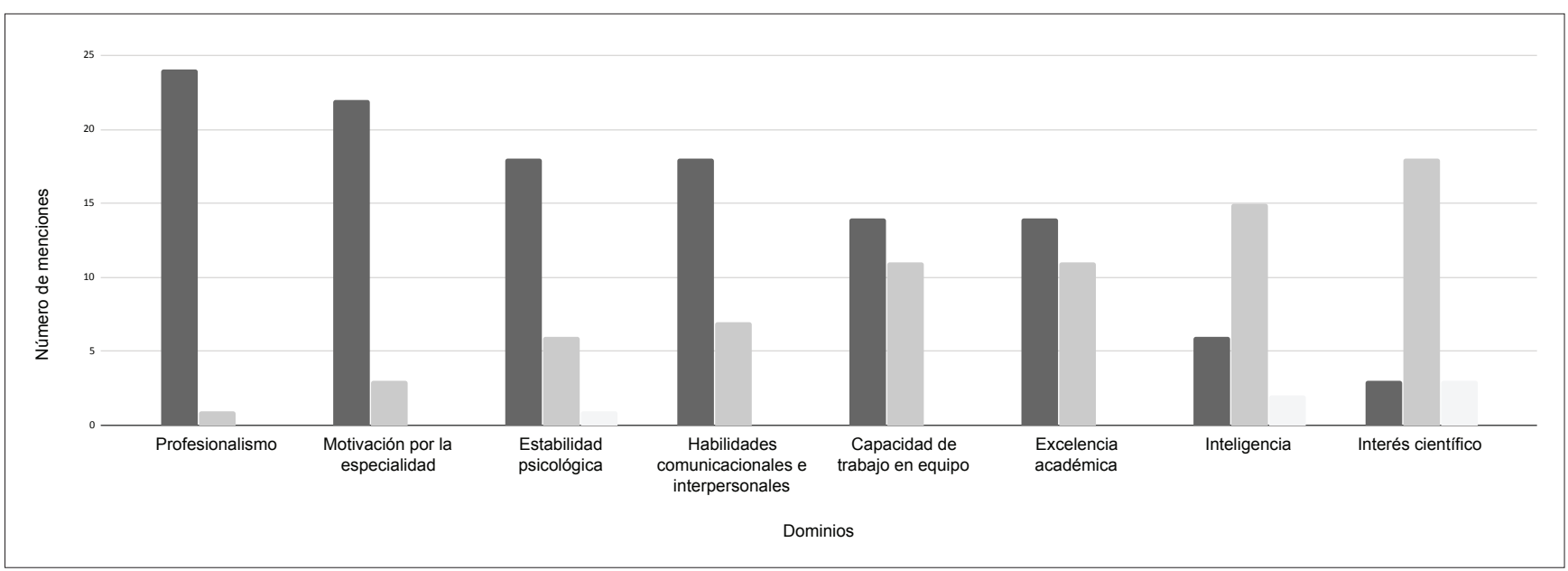

Figura 1: Numero de menciones según grado de importancia del dominio de selección. Gris oscuro: muy de acuerdo (MDA), gris intermedio: de acuerdo (DA), gris claro: en desacuerdo (ED).

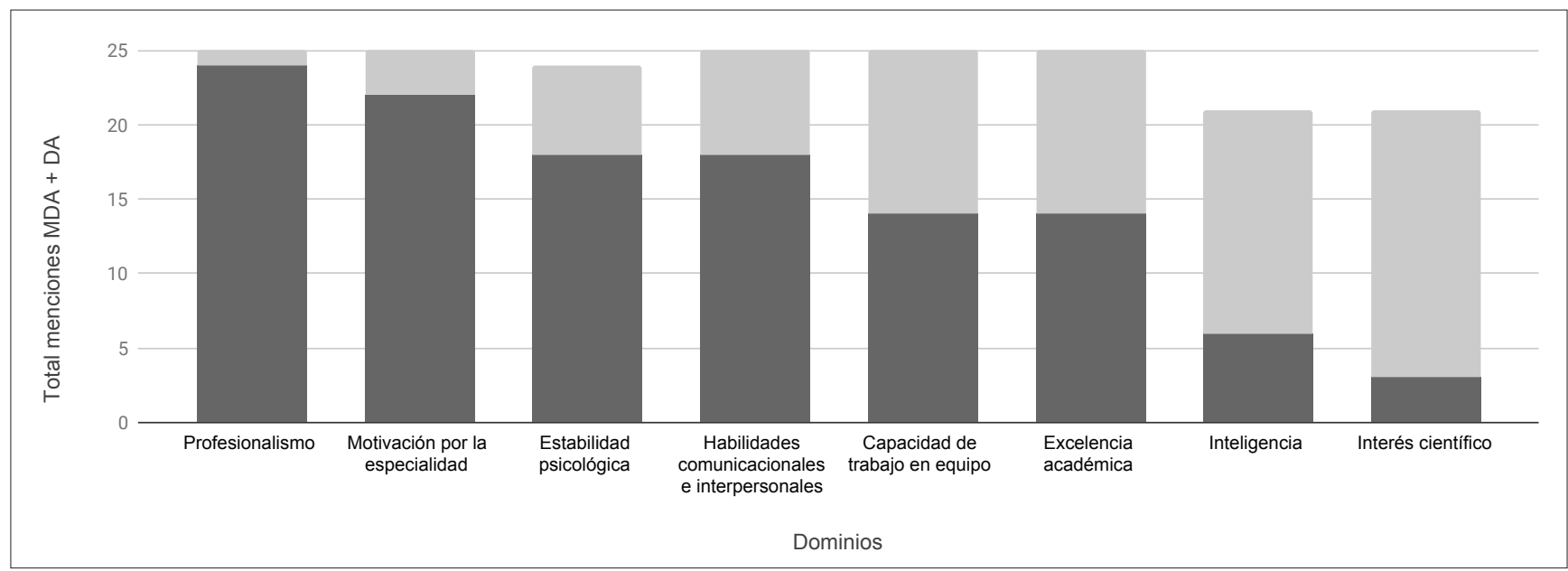

Figura 2: Numero de menciones como grados de acuerdo global (muy de acuerdo + de acuerdo) según grado de importancia del dominio de selección. Gris oscuro: muy de acuerdo (MDA), gris intermedio: de acuerdo (DA).

En la segunda ronda se les preguntó a los encuestados sobre cómo o mediante qué instrumento evaluarían los atributos (dominios) de los postulantes Las principales respuestas tabuladas se centraron principalmente en la importancia de la entrevista personal, sin mencionar mayores características de la misma (95 menciones, 35\% del total de las menciones). Le siguen el número de publicaciones científicas (30 menciones, 11\% del total de las menciones), curriculum vitae (27 menciones, $10 \%$ del total de las menciones), notas de pregrado (25 menciones, $9 \%$ del total de las menciones), actividades relacionadas a la especialidad ( 25 menciones, $9 \%$ del total de las menciones). (Tabla 1).

Al asociar los medios de evaluación de los atributos del postulante con los dominios construidos, se observa que al dominio" profesionalismo" se le busca medir principalmente con la entrevista personal (12 menciones,) y con "carta de recomendación" (11 menciones). En el dominio "motivación por la especialidad", el medio elegido también es la entrevista personal (12 menciones), acompañado del número de "actividades relacionadas con la especialidad" a la cual se postula (ayudantías, asistencia a congresos/ seminarios, pasantías) (10 menciones). Para los dominios "habilidades comunicacionales e interpersonales" $y$ "estabilidad psicológica", nuevamente es la entrevista personal el método más mencionado, con 20 menciones cada dominio. Cabe destacar comparativamente que para el dominio "estabilidad psicológica" el medio de evaluación test o evaluación psicológica es solo 6 veces mencionado en comparación con "entrevista personal" que tiene 20 menciones. Los 3 métodos 
de selección menos considerados por los encuestados, según la prueba nacional de conocimientos de medicina (2 menciones) números de menciones, son "carta personal" (solo una mención), y la "actividades extra programáticas" con solo 2 menciones.

Tabla 1: Principales métodos de evaluación asociado, dominios de selección y número de menciones

\begin{tabular}{|c|c|c|c|}
\hline Método o instrumento de evaluación & Dominios asociados al instrumento y $\mathrm{n}^{\circ}$ de menciones & $\begin{array}{l}n^{\circ} \text { total de menciones } \\
\text { del instrumento }\end{array}$ & $\begin{array}{l}\% \text { del total de } \\
\text { menciones }\end{array}$ \\
\hline Entrevista personal & $\begin{array}{l}\text { Habilidad de comunicación (20) } \\
\text { Estabilidad psicológica (20) } \\
\text { Inteligencia (16) } \\
\text { Profesionalismo (12) } \\
\text { Motivación (12) } \\
\text { Capacidad de trabajo en equipo (10) } \\
\text { Interés científico (5) }\end{array}$ & 95 & $35 \%$ \\
\hline Publicaciones científicas & $\begin{array}{l}\text { Interés científico (15) } \\
\text { Excelencia académica (8) } \\
\text { Motivación (6) } \\
\text { Inteligencia (1) }\end{array}$ & 30 & $11 \%$ \\
\hline Curriculum Vitae & $\begin{array}{l}\text { Excelencia académica (6) } \\
\text { Profesionalismo (3) } \\
\text { Motivación (4) } \\
\text { Inteligencia (2) } \\
\text { Interés científico (5) } \\
\text { Capacidad de trabajo en equipo (5) } \\
\text { Estabilidad psicológica (2) }\end{array}$ & 27 & $10 \%$ \\
\hline Notas de pregrado & $\begin{array}{l}\text { Excelencia académica (18) } \\
\text { Inteligencia (4) } \\
\text { Habilidad de comunicación (2) } \\
\text { Interés científico (1) }\end{array}$ & 25 & $9 \%$ \\
\hline $\begin{array}{l}\text { Actividades relacionadas a la espe- } \\
\text { cialidad }\end{array}$ & $\begin{array}{l}\text { Motivación (10) } \\
\text { Interés científico (7) } \\
\text { Excelencia académica (5) } \\
\text { Profesionalismo (3) }\end{array}$ & 25 & $9 \%$ \\
\hline Cartas de recomendación & $\begin{array}{l}\text { Profesionalismo (11) } \\
\text { Estabilidad psicológica (5) } \\
\text { Capacidad de trabajo en equipo (4) } \\
\text { Habilidad de comunicación (1) }\end{array}$ & 21 & $8 \%$ \\
\hline Test o evaluaciones psicológicos & $\begin{array}{l}\text { Estabilidad psicológica (6) } \\
\text { Inteligencia (3) } \\
\text { Habilidad de comunicación (2) } \\
\text { Profesionalismo (1) }\end{array}$ & 12 & $4 \%$ \\
\hline Entrevista grupal & $\begin{array}{l}\text { Capacidad de trabajo en equipo (5) } \\
\text { Estabilidad psicológica (1) }\end{array}$ & 6 & $2 \%$ \\
\hline Actividades extra programáticas & $\begin{array}{l}\text { Profesionalismo (3) } \\
\text { Habilidad de comunicación (1) } \\
\text { Motivación (1) }\end{array}$ & 5 & $2 \%$ \\
\hline Prueba EUNACOM & Excelencia académica (2) & 2 & $1 \%$ \\
\hline Carta personal & Motivación (1) & 1 & $<1 \%$ \\
\hline
\end{tabular}

\section{Discusión}

El presente estudio permitió conocer las preferencias de un grupo de docentes en relación a los atributos esperables en un postulante a una residencia de Psiquiatría. Los atributos seleccionados fueron agrupados por los autores en 8 dominios o categorías, de los cuales los con mayor preferencia fueron: profesionalismo, motivación por la especialidad, estabilidad psicológica y habilidades comunicacionales e interpersonales. Las categorías "profesionalismo" y "habilidades 
comunicacionales e interpersonales" se encuentran en sintonía con el modelo de desarrollo curricular basado en competencias CANMeds, modelo educacional promovido por la institución a la cual pertenecen los académicos encuestados.

Los dominios "profesionalismo"y "motivación por la especialidad", estuvieron por sobre los dominios de "estabilidad psicológica" y "habilidades comunicacionales e interpersonales". Se podría pensar que los dos dominios más elegidos en este estudio debiesen ser competencias transversales a todo residente, independiente de la especialidad médica, sin embargo, tanto el dominio de "estabilidad psicológica" como el de "habilidades comunicacionales e interpersonales" se supondría que podrían ser atributos más requeridos y necesarios en la especialidad de Psiquiatría en comparación con otras especialidades. En este caso esto no ocurre. Al respecto, se podría plantear que quizás los docentes encuestados pudieran creer que el dominio "habilidades comunicacionales e interpersonales" se podría ir adquiriendo durante la formación por medio del entrenamiento, y por ende no ser prioritario para el inicio de un programa de residencia. En tanto, el profesionalismo del residente impresiona ser una característica esencial desde el inicio del entrenamiento.

Con respecto a "estabilidad psicológica", se podría pensar que es determinante para una formación de Psiquiatría y que debería estar en términos de prioridad sobre el dominio "motivación por la especialidad", sin embargo, esto no ocurre en este estudio. Quizás se necesiten definiciones más precisas cuando se estudien estos dominios, o bien, los encuestados consideraron la estabilidad psíquica del postulante casi asegurado por ser un médico ya formado.

En este estudio, cuando se quiso evaluar el dominio habilidades "comunicacionales e interpersonales" y "estabilidad psicológica", dominios a nuestro parecer relevantes en la formación en Psiquiatría, los resultados mostraron que los encuestados prefirieron el instrumento entrevista personal antes que el uso de test psicológicos. Se podría pensar que la inexistencia de pruebas psicológicas específicas para aplicarles a los postulantes en relación a la medición de estas habilidades estimule optar por la entrevista directa, o bien, los docentes evaluadores en estos casos confían más en su "olfato clínico" que en los test. Las pruebas que se aplican hoy en día en algunos centros formadores miden más bien rasgos de personalidad o capacidad intelectual de modo general, ambos atributos fueron categorizados como de menor importancia en este estudio.

Se conoce que las entrevistas personales son una herramienta útil para evaluar habilidades interpersonales y de comunicación en procesos de selección (Shiroma \& Alarcón, 2010; Strand, 2011). Los resultados obtenidos en el presente estudio respecto al instrumento "entrevista personal" se condicen con algunos resultados disponibles en la literatura respecto a que esta herramienta es muy recurrida por los profesionales al momento de seleccionar a otros profesionales postulantes a residencias (Dubovsky et al., 2008). Sin embargo, se debe tener presente que las entrevistas personales en su conjunto han demostrado poco valor predictivo al momento de relacionarla con el rendimiento del residente durante su formación posterior y parece ser solo de mayor utilidad cuando es usada como herramienta para evaluar el interés del postulante por la especialidad (Dubovsky et al., 2008; Dawkins et al., 2005), o bien cuando ciertas características personales que se esperan del postulante, por ejemplo, motivación, actitud positiva y relaciones personales satisfactorias, quedan pauteadas y estructuradas por el comité docente (Brothers \& Wetherholt, 2007). De continuar usándolas, las entrevistas personales se deberían asociar a otros instrumentos objetivos de evaluación (Brothers \& Wetherholt, 2007), o bien hacerlas sin conocer previamente los antecedentes académicos del postulante, evitando el sesgo (Illahi, 2017).

Queda pendiente, sin embargo, y como línea de investigación futura, comprobar la asociación de los dominios sugeridos por los participantes con el desempeño y éxito del proceso formativo de los postulantes seleccionados, evaluando de cada uno su eficacia predictora.

El presente estudio presenta algunas limitaciones. Como se expuso desde un comienzo, es un estudio realizado sobre un grupo de docentes de Psiquiatría de una única entidad formadora. Por lo tanto, los resultados obtenidos no son extrapolables a las demás especialidades médicas de la institución, como tampoco a otras instituciones formadoras. Se añade a lo anterior limitaciones propias de la utilización del método de estudio Delphi, ya que los resultados obtenidos en las distintas fases varían según cómo se formulen las preguntas y como la búsqueda de consenso limita la creación de nuevas respuestas, y en consecuencia la creación de nuevos conceptos o dominios.

\section{Conclusiones}

La selección del mejor candidato para una residencia en Psiquiatría es un desafío tanto para el equipo docente como para la institución formadora. Es en los criterios o los dominios considerados esenciales para la formación médica, donde se plasma la misión misma de la institución formadora. Disponer de un conjunto de características definidas y conocidas que se buscan en el candidato a residente, permite definir el perfil y el sello particular del especialista que se desea formar. Por otro lado, disponer de instrumentos específicos y validados en la selección de residentes permite que aquel perfil permanezca en el tiempo y no se modifique con cambios transitorios. 
Un buen método de selección debería en lo posible pronosticar un mejor rendimiento del residente durante el programa, menos remediaciones y mejor desempeño futuro.

Agradecimientos: docentes y académicos del Departamento de Psiquiatría de la Pontificia Universidad Católica de Chile.

\section{Referencias}

Agarwal V., Bump G., Heller M., Chen LW., Brnasteter B., Amesur N., et al. (2018). Do residency selection factors predict radiology resident performance? Acad Radiol, 25, 397-402.

Berner ES., Brooks C. \& Erdmann JB. (1993). Use of the USMLE to select residents. Acad Med, 68, 753-759.

Brothers TE. \&Wetherholt S. (2007). Importance of faculty interview during the resident application process. J Surg Edu, 64, 378-385.

CanMEDS. (2019). The Royal College of Physicians and Surgeons of Canada. Accedido en http://www.royalcollege.ca/rcsite/canmeds/ canmeds-framework-e el 22 de mayo 2019.

Clouet-Huerta D., González B. \& Correa K. (2017). Especialización médica en Chile: tipos, mecanismos y requisitos de postulación. Una actualización de los procesos para los médicos generales. Rev Med Chile, 145, 1454-1462.

Dawkins K., Ekstrom R., Maltbie A. \& Golden R. (2005). The relationship between psychiatry residency applicant evaluations and subsequent residency performance. Acad Psychiatry, 29, 69-750.

Dubovsky S., Gendel M., Duvosky A., Levin R., Rose J. \& House R. (2008). Can admissions interviews predict performance in residency? Acad Psychiatry 32, 498-503.

Escuela de Medicina PUC. (2019). La nueva forma de enseñar medicina centrada en las competencias - Escuela de Medicina. Accedido en https://medicina.uc.cl/noticias/la-nueva-forma-de-ensenar-medicina-centrada-en-las-competencias_el 5 de junio 2019.

Egol KA., Collins J. \& Zuckerman JD. (2019). Success in orthopedic training: resident selection and predictors of quality performance. J Am Acad Orthop Surg. 19, 72-80.

Green M., Jones P. \& Thomas X. (2009). Selection criteria for residency: results of a national program directors survey. Acad Med, 84, 362-367.

Harfmann K. \& Zirwas M. (2011). Can performance in medical school predict performance in residency? J Am Acad Dermatol. 65, 1010-1022.
Hamdy H., Prasad K., Anderson MB., Schrpbier A., Williams R., Zwierstra R., et al. (2006). BEME systematic review: predictive values of measurements obtained in medical schools and future performance in medical practice. Med Teach. 28, 103-116.

Hartman ND, Lefebvre CW. \& Manthey DE. (2019). A narrative review of the evidence supporting factors used by residency program directors to select applicants for interviews. J Grad Med Educ, 11, 268-273.

Illahi Y., Alkhoury G., Khan Z., Barnett W. \& Assaly R. (2017). Panel interview for internal medicine selection: pros and cons. Int J Med Educ, 8, 59-60.

Jaramillo L. \& Martín E. (2012). Evaluación integral de residentes de psiquiatría: un complemento del proceso de admisión al programa. Rev Colomb Psiquiat. 41, 104-112. Disponible en: http://www.scielo.org.co/ scielo.php?script=sci_arttext\&pid=S0034-74502012000500011\&lang=es. Acceso: 29 de mayo 2019.

Kenny, S., Mclnnes, M. \& Singh, V. (2013). Associations between residency selection strategies and doctor performance: a meta-analysis. Med Educ 47: 790-800.

Linstone, H. (2002). The Delphi Method: Techniques and Applications. H. Linstone and M. Turoff (Eds.). Accedido en https://web. njit.edu/ turoff/pubs/delphibook/index.html el 15 de junio 2019.

Lobato, R., Lagares, A., Villena, V., García J., Jiménez-Roldan L., Munarriz P., et al. (2015). La selección de los graduados en Medicina para los puestos de residencia. Estudio comparativo de los métodos usados en diferentes países. Neurocirugía, 26, 3-12.

Lorusso N. \& Gonzalez B. (2013). Variabilidad de los procesos selectivos para el acceso a las especialidades médicas en Europa: entre convergencia y divergencia. FEM: Revista de la Fundación Educación Médica, 16, 215-218. Accedido en http://scielo.isciii.es/ scielo.php?script=sci_arttext\&pid=S2014-98322013000600005_el 25 de mayo 2019.

MINSAL (2019). Concurso 2019 para médicos y odontólogos. Accedido en Https://www.minsal.cl/concurso-medicos-odontologos-2019 el 21 junio 2019.

Prober C., Kolars J., First L. \& Melnick D. (2016). A plea to reassess the role of United States Medical Licensing Examination Step 1 scores in residency selection. Acad Med, 91:12-15.

Shiroma P. \& Alarcon R. (2010). Selection factors among international medical graduates and psychiatric residency performance. Acad Psychiatry, 34, 128-131. 


\section{Pacheco et al.}

Smilen S., Funai E. \& Bianco A. (2001). Residency selection: should interviewers be given applicants' board scores? Am J Obstet Gynecol, 184 (3), 508-513.

Stephenson-Famy A., Houmard B., Oberoi S., Manyak A., Chiang S. \& Kim S. (2015). Use of the Interview in Resident Candidate Selection: A Review of the Literature. J Grad Med Educ, 7, 540-548. Accedido en https://www.ncbi.nlm.nih.gov/pmc/articles/PMC4675409/ el 15 de junio 2019.

Strand EA., Moore E. \& Laube DW. (2011). Can a structured, behavior-based interview predict future resident success? Am J Obstet Gynecol, 204, 446.e1-446.e13.
Wagner J., Schneberk T., Zobrist M., Hern HG., Jordan J., Boysen-Osborn M. et al. (2017). What predicts performance? A multicenter study examining the association between resident performance, rank list position, and United States Medical Licensing Examination Step 1 Scores. J Emerg Med 52, 332-340.

Wagoner N, Suriano J. \& Stoner J. (1986). Factors used by program directors to select residents. J Med Educ. 61, 10-21.

Wagoner N. \& Suriano R. (1992). Recommendations for changing the residency selection process based on a survey of program directors. Acad Med, 67, 459-465. 